Article

\title{
Development of an IoT-Based Health Promotion System for Seniors
}

\author{
Chia-Hui Liu ${ }^{1, *}$ and Jih-Fu Tu ${ }^{2}$ \\ 1 Department of Applied Mathematics, Chinese Culture University, Taipei 11114, Taiwan \\ 2 Department of Electrical Engineering, Lunghwa University of Science and Technology, \\ Taoyuan City 33306, Taiwan; tujihfu@gmail.com \\ * Correspondence: ljh34@ulive.pccu.edu.tw
}

Received: 18 July 2020; Accepted: 24 October 2020; Published: 28 October 2020

\begin{abstract}
With a reducing fertility rate and the increase in life expectancy in Taiwan, the changing structure of the population has pushed Taiwan into an aged society. The demand for elderly care environments is rapidly increasing. However, to prevent an illness for the elderly is better than to treat it. Monitoring of an individual's health is required as a periodic activity. Using wireless technology combined with physiological measurement techniques and home care equipment can assist elderly individuals to promote health and the health care service at home. In this research, a wireless sensor network has been adopted to develop context aware health promotion for an elderly-care system. The system is divided into three subsystems: the IoT-based physiological information subsystem, the context awareness-based service subsystem, and the elderly nutrition diet and health promotion subsystem. In this system, users also can select an Aging Diet Module or an Aging Exercise Module in our app. After the user selects the Aging Diet Module, the system will record and give suggestions. Moreover, the system can integrate long-term elderly diet and exercise records, and then, assist elderly to complete personal nutrition assessment and health management. The research goal is to construct a health promotion system utilizing IoT technology. In this paper, the system's functions and implementation approaches are introduced.
\end{abstract}

Keywords: elderly care; wireless sensor network; context awareness; health promotion

\section{Introduction}

Issues based on advances in medical technology coupled with science and technology and economic and work pressures have resulted in decreasing fertility rates in Taiwan in recent years and the average life expectancy of its citizens to continually increase. In the definition by the World Health Organization (WHO), a county is regarded as an aging society if their population aged above 65 years old has exceeded $7 \%$. Taiwan has become an aging society, since its elderly population already reached $7.1 \%$ of the total population in 1993. According to the population statistics published from the Ministry of the Interior in Taiwan, the elderly population is predicted to reach $20 \%$ of the total population in 2025 [1]. WHO experts also stated the importance of a healthy lifestyle at every stage of life; since 2001, health promotion strategies focused on the elderly started to grow in numbers. Before that, people commonly assumed that there was no need for the elderly to participate in health promotion activities as it might be too late to change their lifestyle. Nowadays, the elderly is the addressee of health promotion activities. Health promotion targeted at older people differs significantly from that targeted at younger people because the health condition of older people is generally less than perfect $[2,3]$. Therefore, health promotion strategies for the elderly generally have the basic aims of maintaining or improving self-care [4]. 
With technological progress, information technologies can help the progress of maintaining or improving self-care by exploiting the most advanced technologies of the Internet of Things (IoT), such as Radio Frequency Identification, wearable electronics, Wireless Sensor Networks, and Artificial Intelligence. This research constructs a health care promotion system utilizing IoT technology which is to employ wireless sensing network technology to collect physiological information of elderly people, thereby providing a comprehensive and integrated elderly care system. To prevent an illness for the elderly is better than to treat it, so monitoring an individual's health is required as a periodic activity. The system's objective is to maintain or improve self-care toward the elderly's health promotion. It can help to address one issue of sustainable health: well-being of the elderly. It can also reduce unnecessary mobility for the elderly (e.g., to visit doctors), for sure helping to transform to more sustainable mobility.

This system is mainly divided into three subsystems, namely: (1) IoT-based physiological information subsystem: a wireless sensor monitoring physiological information subsystem will collect elderly physiological data and information to provide real time and appropriate home health care. (2) Context awareness-based service subsystem: by providing context awareness services for living situations, it improves the quality of life of elderly people at home. (3) Elderly nutritional diet and health promotion subsystem: a service subsystem for elderly nutritional diet and health promotion. The senior may suffer from physical decline such as decreased chewing ability or digestive ability due to aging, which makes such individuals more likely to have diseases. By improving the eating habits of the elderly, further promoting the absorption of appropriate nutrients, and increasing physical strength, disease prevention may be augmented. The user-oriented system is programmed for the elderly, allowing users to enter personal data directly into their personal smartphones. This nutritional diet and health promotion subsystem can recommend to the elderly exclusive daily nutritional diet recommendations based on personal information. After the user selects the Aging Diet Module, the system will record and give suggestions. Through the Aging Exercise Module, this subsystem can provide users with recommended exercise frequency, intensity, and content. In other words, the system can integrate long-term elderly diet and exercise records, and then, assist the elderly to complete personal nutrition assessments and health management.

\section{Related Works}

Due to advances in technology, there have been many related researches on IoT technology, home care system application development, context awareness services, and elderly nutritional diet and health promotion.

\subsection{Internet of Things}

In an aging society, it is an important goal to use technology to complete local elderly and home health care or health promotion $[5,6]$. The application of the IoT and wearable technology could improve the quality of life for elderly, decrease strain on the health promotion system, and make the system operational cost less [7]. Users actively measure blood pressure, blood glucose, weight, electrocardiograms, and even various health signals such as Activity of Daily Living (ADL) at home [8]. It commonly uses wireless networks to transmit information, and wireless sensors to collect information. The sensor equipped with the wireless transceiver forms a WSN, which can provide a more comfortable living environment for the user, allowing the user to move freely without wearing a sensor device that needs to be connected with a wired cable, providing a more convenient life [9]. On the other hand, the use of wireless communication networks can significantly reduce wiring costs. When the elderly is receiving care, the five basic programs proposed by the General Assembly of the United Nations in 1991 (UN, 1991) [10] must be considered: independence, participation, care, self-fulfillment, and dignity.

The wireless sensor network concept mentioned was derived from UC Berkeley's Smart Dust research project. A wireless sensor network system [1] refers to a technology that can integrate sensing, 
computing, and wireless networks. Wireless sensor networks have several characteristics, including low cost, short distance required for wireless transmission, small size, low power consumption, fault tolerance, and self-configuration. The basic principle of its operation is to deploy many sensors in the area that needs to be sensed in order to collect external environmental data, such as humidity, temperature, lightness, and other environmental information. Afterwards, the sensors will use the self-organization protocol to connect the sensors into a communication network. In the next step, the data are transmitted to the data collector through the one-hop or multi-hop transmission method, and then, the data are transmitted to the manager or user for reference by the collector. Benefiting from the development of microdevices and embedded technology, small electronic devices can be embedded with sophisticated multi-functions such as precise sensing, computing, and communication. This type of wireless sensor can not only detect and collect external environmental data, but also analyze and process the collected data in-depth.

\subsection{Application and Development of Health Care}

Computing technology has become commonplace in today's health care and ever-present advances in technology continue to influence the medical industry. The use of a wireless sensor network is one such recent advancement. It allows computing technology to become mobile. This mobility has significantly increased the speed at which information can be accessed. It applies various sensors to help collect users' physiology signs and information.

This information is distributed to the data receiver via wireless transmission. Then, the information is transmitted to the external network through a wireless router, and then, to the central server of a specific institution such as a care center or hospital via the Internet [11]. It commonly uses wireless networks to transmit information, and wireless sensors to collect information.

There were some works in the field of user health and medical condition issues using the IoT to promote users' health. A health monitoring system has been developed [12] that can monitor basic symptoms of a patient like heartbeat, percentage of oxygen saturation, and body temperature in an IoT network. For this purpose, the system used heartbeat, oxygen saturation, temperature, and eye blink sensors as capturing elements and Arduino-UNO as a processing device. The system has already been developed but no specific performance measures have been analyzed for users. Acharya et al. [13] introduced health care monitoring in an IoT environment. The system monitored basic parameters of user health like heartbeat, ECG, and body temperature. It collected data from sensors and transmitted them to a server. However, it has no visualized interfaces for common users. Milon Islam et al. [14] proposed a smart health care system in an IoT environment. It monitors a patient's physiological signs and the room conditions where the patient is. The patient's condition can immediately be transmitted to medical staff, where they can process and analyze the current situation of the patient.

\subsection{Context Awareness Service for Elderly}

The idea of Context Awareness was first proposed by Schilit and Theimer [15] in 1994. This core theory refers to collecting information of the user's environment through sensors, and then, the backend judges the scenarios and provides relevant information based on the current environmental information. Context awareness has the following four representative characteristics: automation, comprehensiveness, immediacy, and customization [16]. These scenarios fall into three main categories:

1. Computing scenarios, such as network interfaces, network bandwidth, CPU computing power, or other related computing resources.

2. User scenarios. Refers to the user's current location, usage habits, or current user behavior.

3. Actual environment scenarios. Mainly refers to the actual environmental conditions, such as temperature, light, and sound. The context awareness function for the elderly must support the following three items: (1) Presentation of information and services to a user. (2) Automatic execution of a service for a user. (3) Tagging of context to information to support later retrieval. 
Another context awareness application is fall detection. The University of California, Berkeley proposed a wearable fall detection and warning device in a research report [17]. The research method adopted an acceleration sensor to detect the acceleration values in various directions accompanying the fall. The hardware of this device can be roughly divided into microcontrollers, signal sensors, alarms, and positioning, etc. Among them, the microcontroller part is responsible for acceleration signal acquisition, signal analysis, and logical judgment of the fall condition, and actively warns when the fall condition is recognized. In addition, the sensor can detect and collect the temperature, humidity, air quality, etc., in the user's environment to construct a perfect situational awareness system.

\subsection{Elderly Nutritional Diet and Health Promotion}

Due to the progress of medical systems, the population structure of each place has gradually transformed into an aging society. Because of aging, older people are facing physical decline, such as decreased chewing ability, appetite, and digestive ability, which in turn reduces their physical strength and weight, and eventually, leads to more susceptibility to disease. To improve the diet of the elderly, it can start by promoting the absorption of nutrients, thereby enhancing physical strength and activity. In addition, if the elderly can exercise properly, it will help strengthen their immune system, expel toxins from the body, and maintain normal limbs and cognitive function. In this way, the incidence of illness and becoming bedridden can obviously be reduced, and the health insurance expenses of the elderly can be further reduced [18]. The nutritional diet of the elderly can be divided into the following three categories:

1. Easy to eat: Due to the weak chewing or swallowing ability of older people, some older people may have tooth loss or decreased saliva secretion may reduce their digestive ability. This situation makes it difficult for the elderly to eat even if they want to. In response to such problems, the elderly's food is often made into a mild diet that is chopped or liquid. Making delicious and nutritious food with the texture of food that can be swallowed by the elderly has always been a major challenge to meet the nutrition balance.

2. Insufficient calories: Many elderly people have weakened chewing ability or decreased appetite, making their taste dull. Therefore, elderly people may be unwilling to eat or have trouble cooking, and often use cereal, porridge, and pickled food as their main meal. Elderly people often lack enough vegetables, fruits, and dairy products. Therefore, when the appetite of the elderly becomes worse, drinking water or soup while eating should be avoided, so as not to be too full. In addition, although porridge is easier to digest than white rice, it is also easier for older people to feel full, so it is recommended that elderly people change to frequent small meals.

3. Consumption of balanced nutrition: Although some seniors believe they have a healthy diet, they often choose to be vegetarians because they believe in secret recipes, or some elderly people are too worried about their chronic diseases and overly restrict their diet, which may eventually lead to malnutrition.

\section{The IoT-Based Health Promotion System}

\subsection{The IoT Based Health Promotion System Architecture}

The IoT technology used in this paper provides a comprehensive, integrated care system based on context awareness of elderly-oriented health care promotion systems. The system is mainly divided into three subsystems, as shown in the Figure 1:

1. The IoT-based physiological information subsystem;

2. The context awareness-based service subsystem;

3. The elderly nutrition diet and health promotion subsystem.

This research is not only based on collected elderly physiological data and information to provide timely and comprehensive home health care, but also to increase the safety and convenience of the 
home living environment through context awareness services. For example, the system is dedicated to preventing the elderly from falling. By actively sensing the surrounding environment and warning in advance, it reduces the possibility of falls and avoids the need for subsequent fall notification or treatment. In addition, the system also provides context awareness services for living environments to improve the quality of life for elderly people.

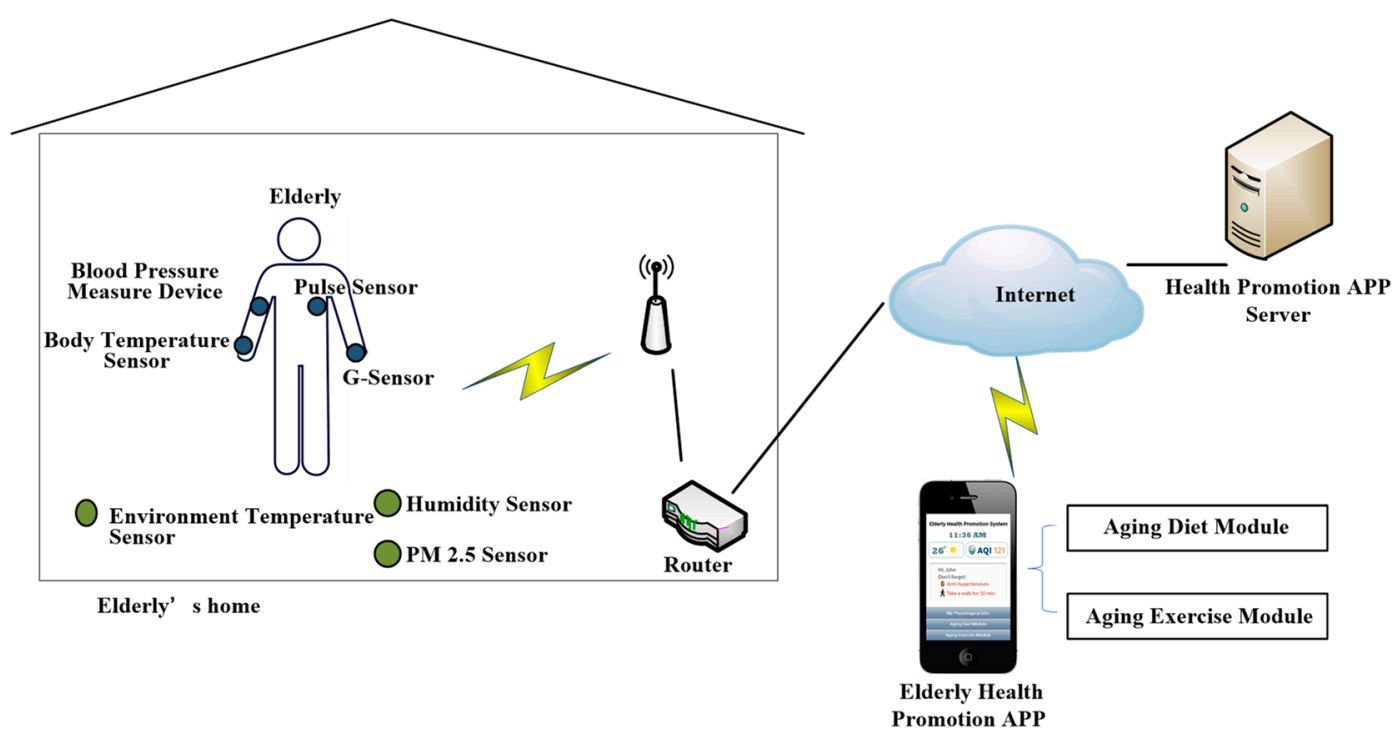

Figure 1. The IoT-based Health Promotion System architecture.

Most importantly, the system is elderly-oriented, allowing users to enter personal data directly on their personal smartphones, and the system provides dedicated daily nutritional recommendations based on personal data. After the user selects the Aging Diet Module, the system will record it. Then, the system will suggest the calories to be consumed and provide the user with the recommended amount of exercise through the Aging Exercise Module. This system can integrate long-term elderly diet and exercise records and assist the elderly to complete personal nutrition assessments and health management.

\subsection{Three Subsystems}

\subsubsection{The IoT Based Physiological Information Subsystem}

The framework presented in Figure 1 demonstrated that the cared ones will wear a sensor device to capture physiological information, the base station will send a request to the sensor, and then, the wireless sensor will transmit the collected physiological information to the base station. This information will be sent to the wireless router deployed in the home through the wireless sensor worn on the body and will be forwarded to the information transmitter from station to station in a multi-hop manner. Finally, the physiological information can be sent to the health promotion app server. The health promotion app server will regularly upload the patient's physiological information to the server in the care center through the Internet. Professional health care personnel can use the comprehensive physiological information provided by this system to review and analyze the data to determine whether the cared ones need assistance, or related health reminders. The health promotion server is also responsible for assisting with the provision of context sensing services. It will collect relevant sensing signals sent by the sensor device to determine the current situation of the user and further provide corresponding services.

In the system, the three-subsystem server was constructed in the health promotion app server and integrated into one mobile phone application. Meanwhile, the relative sensors were set up to surround users or to be put on their body. Seniors can review their physiology data through the mobile phone app. 


\subsubsection{The Context Awareness Service Subsystem}

The subsystem is divided into six modules, as described below:

1. Context awareness acquisition module: The context awareness module uses a variety of sensors to comprehensively collect all context information. This means that the user's environment, such as people, events, timing, places, and other related information, will activate the signal collection device in this study. At a predetermined time, the sensor will send the collected physiological signals to the homecare server and use the scenarios reasoning module to perform inference. Finally, the scenario knowledge will be stored in the scenario knowledge library.

2. Context knowledge module: This is used to store contextual knowledge, including information about prescriptions for the cared ones, medication time, medication records, toilet frequency, blood pressure changes, temperature changes, and more.

3. Context reasoning engine module: Uses rules to further determine the mode and infer the current situation of the elderly.

4. Service guidance module: This module is service-driven and starts related services based on the results of the context reasoning engine module. For example, the elderly in Taiwan often suffer from certain physical diseases due to aging, and these symptoms may require long-term medication. However, some elderly may forget to take their medicine because of some other factors. The device will record this service itinerary. When the medication time is up, the service guidance module will automatically remind the user to take their medicine.

5. Sensing device module: This is mainly responsible for providing different interfaces to connect with various care devices that measure physiological information.

6. Message module: Responsible for processing, receiving, and transmitting messages, mainly based on the types of sensing signals, such as general routine physiological measurement information, emergency signals, equipment error signals, etc., and then, send corresponding messages through the home-care server.

Those context awareness sensors were applied in the system, such as temperature, air conditioning, and G-sensors. This is a small, thin, low-occupancy three-axis accelerometer, suitable for mobile device applications to build our system. The G-sensor is also called an accelerometer, which is different from a gyroscope that provides heading information. Its detection method of the sensor is to simultaneously detect the movement and value of the three axes and provide information about changes in acceleration. That information can be used to detect whether the user falls. In addition, the G-sensor is a small, thin, low power consumption three-axis accelerometer, suitable for mobile device applications. The elderly must wear it as a wearable device. The G-sensor is embedded in this system. The user wears the mobile device with sensors. Figure 1 shows the context aware sensors of the system, including: ambient temperature sensor, humidity sensor, and PM 2.5 sensor. These data will be collected and transmitted to the health promotion app server. If the data exceed the threshold, the server will push notifications to the mobile phones of the elderly who have installed our elderly health promotion app. In addition, when the system detects a fall, it sends alarm message to notify the user's relatives using the phone number preset in the mobile app.

\subsubsection{The Elderly Nutrition and Health Promotion Subsystem}

In this subsystem, users can select the Aging Diet Module or Aging Exercise Module in a special app. This Aging Exercise Module will provide information based on the above content and give exclusive daily nutritional diet recommendations based on the relevant personal information of the elderly person. Meanwhile, the system also provides users with suggested exercise amounts and content through the Aging Exercise Module. Considering the physical strength of the elderly, the research used the Frequency, Intensity, Type, Time (F.I.T.T.) principle of exercise to give advice. Health promotion campaigns suitable for the elderly should consist of four factors: (1) exercise 
frequency, (2) exercise intensity, (3) exercise duration, and (4) exercise type. The detailed explanations of the above four items are as follows [19]:

1. Exercise frequency: Elderly exercise focuses on increasing the frequency (recommended 5-7 times a week). Regular exercise can increase the dependence of exercise, and at the same time, transform physical activity into a part of the daily life of the elderly, making it a lifestyle, which is more in line with the benefits of health promotion.

2. Exercise intensity: It is necessary to give physical fitness and exercise advice to seniors with different physical functional conditions and physiological characteristics. The intensity control is very important. For older people who are slow-moving, it is recommended to perform slow movements without requiring too much exercise intensity. The main purpose is to exercise muscles.

3. Exercise duration: The duration of continuous exercise for seniors must also be adjusted according to different physical conditions. If their body function is limited to doing 2 times a day, $5-10 \mathrm{~min}$ each time is also a good suggestion; the focus is on maintaining their exercise habits.

4. Exercise type: Establish different sports according to the user's physical condition, such as: walking slowly, stepping, stretching exercises, etc.

\section{The Elderly Health Promotion System Implementation}

The system implementation was introduced as follows. The elderly health promotion system is designed for mobile equipment. In addition, we established a database to keep all measured data and provide to the user inquiring. When the system app records the elderly person's data, it will determine whether the data exceed the normal range. Additionally, the records will transmit to the server to store into the MySQL database and are offered to managers or health caregivers. The back-end elderly health promotion system uses the Apache server and the MySQL database records and backs up the elderly's information and physiological data.

\subsection{The Experiment of the System}

The participants consisted of 30 persons from New Taipei City of Taiwan to use this research system. They were between 62 and 68 years old. Table 1 is the characteristics of the participants. There were 16 men averagely at the age of 67.5 years old, weight $72.1 \mathrm{~kg}$, and height $171.5 \mathrm{~cm}$ (the corresponding standard deviations are 1.61, 8.31, and 4.17). There were 14 women averagely at the age of 66.3 years old, weight $61.6 \mathrm{~kg}$, and height $162.7 \mathrm{~cm}$ (the corresponding standard deviations are $2.19,9.12$, and 4.42). In this research, those users mainly assisted us to test and adjust the system and provided useful feedback suggestions. In total, $73 \%$ of participants are satisfied with the system and they think it would be helpful for promoting health. However, $31 \%$ of users needed their relative's assistance when operating the system.

Table 1. Characteristics of the participants.

\begin{tabular}{ccccc}
\hline & Man_Mean & M_SD & Woman_Mean & W_SD \\
\hline Age & 67.5 & 1.61 & 66.3 & 2.19 \\
Weight $(\mathrm{kg})$ & 72.1 & 8.31 & 61.6 & 9.12 \\
Height $(\mathrm{cm})$ & 171.5 & 4.17 & 162.7 & 4.42 \\
\hline
\end{tabular}

Those elderly can use this system to record their food and drink. It also provides the user information about their surroundings. It can remind the user to exercise every day. It helps the elderly know the nutrients which are suitable for them. The system offers a physiological data feedback curve for users to understand their health conditions and avoid the occurrence of diseases. Figure 2 is the client interface of the elderly health promotion system. The elderly can see the temperature and air quality of his local area. At the same time, the system will also display information on the 
user dashboard, such as medication reminders, exercise reminders, etc. The user also can access the personal physiological information page to view various physiological data and trend chart.
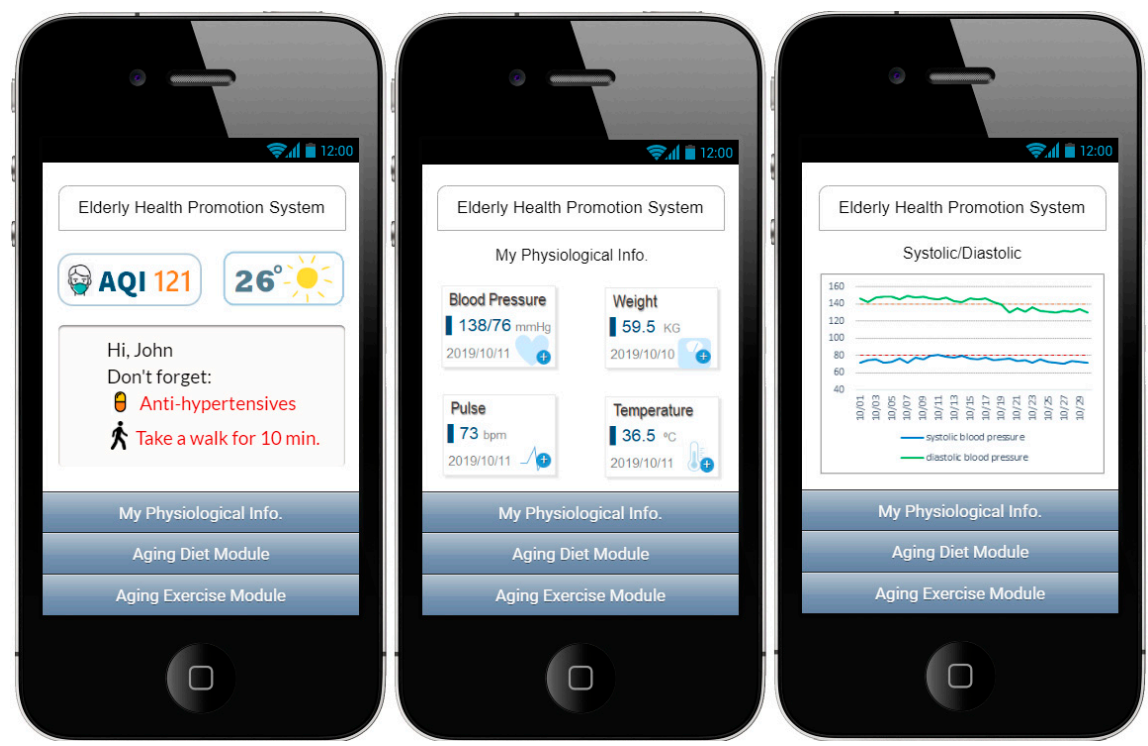

Figure 2. The client interface of elderly health promotion system.

Air Quality Index (AQI) is divided into six levels from good (green) to hazards (brown), using the $\mathrm{AQI}$ as the quality forecast standard. For example, when the color is orange, it means that allergic groups or children and the elderly should be more careful, and they are best to wear a mask. In the system, the AQI value will be displayed in corresponding colors based on different data. On the other hand, the icon on the left also shows different meanings. In this case, the icon on the left in the Figure 2 indicates that the elderly person is recommended to wear a mask. The detailed meanings of the AQI values are as follows:

1. 0-50: Good. Air quality is considered satisfactory.

2. 51-100: Moderate. Air quality is acceptable; however, there may be a moderate health concern for people who are unusually sensitive to air pollution.

3. 101-150: Unhealthy for Sensitive Groups. Members of sensitive groups may experience health effects.

4. 151-200: Unhealthy. Everyone may begin to experience health effects.

5. 201-300: Very Unhealthy. Health warnings of emergency conditions. The entire population is more likely to be affected.

6. 300+: Hazardous. Health alert: everyone may experience more serious health effects.

Moreover, the system management or caregiver who has authority to access the user's information can review the elderly's physiological data and the trend chart for the past month, as in Figure 3.
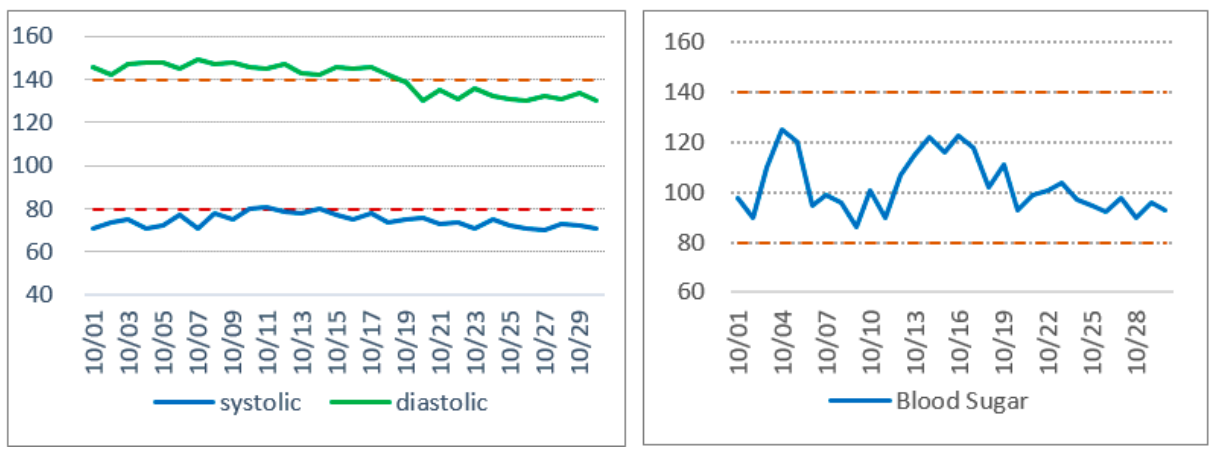

Figure 3. User's physiological data visualization. 


\subsection{The Elderly Health Promotion Application Interface}

If the elderly has access to quality health care and improves their daily diet, this application can promote health and reduce the chance of becoming sick and bedridden. This study designed a health promotion application to provide an Aging Diet Module, designed it based on the elderly, and gave exclusive daily nutritional diet recommendations based on the relevant personal information of the elderly. In addition, the system also provided suggested exercise amounts to users through the Aging Exercise Module. This section is designed to be easy to use and easy to read. This function can be divided into two modules. The following is the app system flow chart (Figure 4).

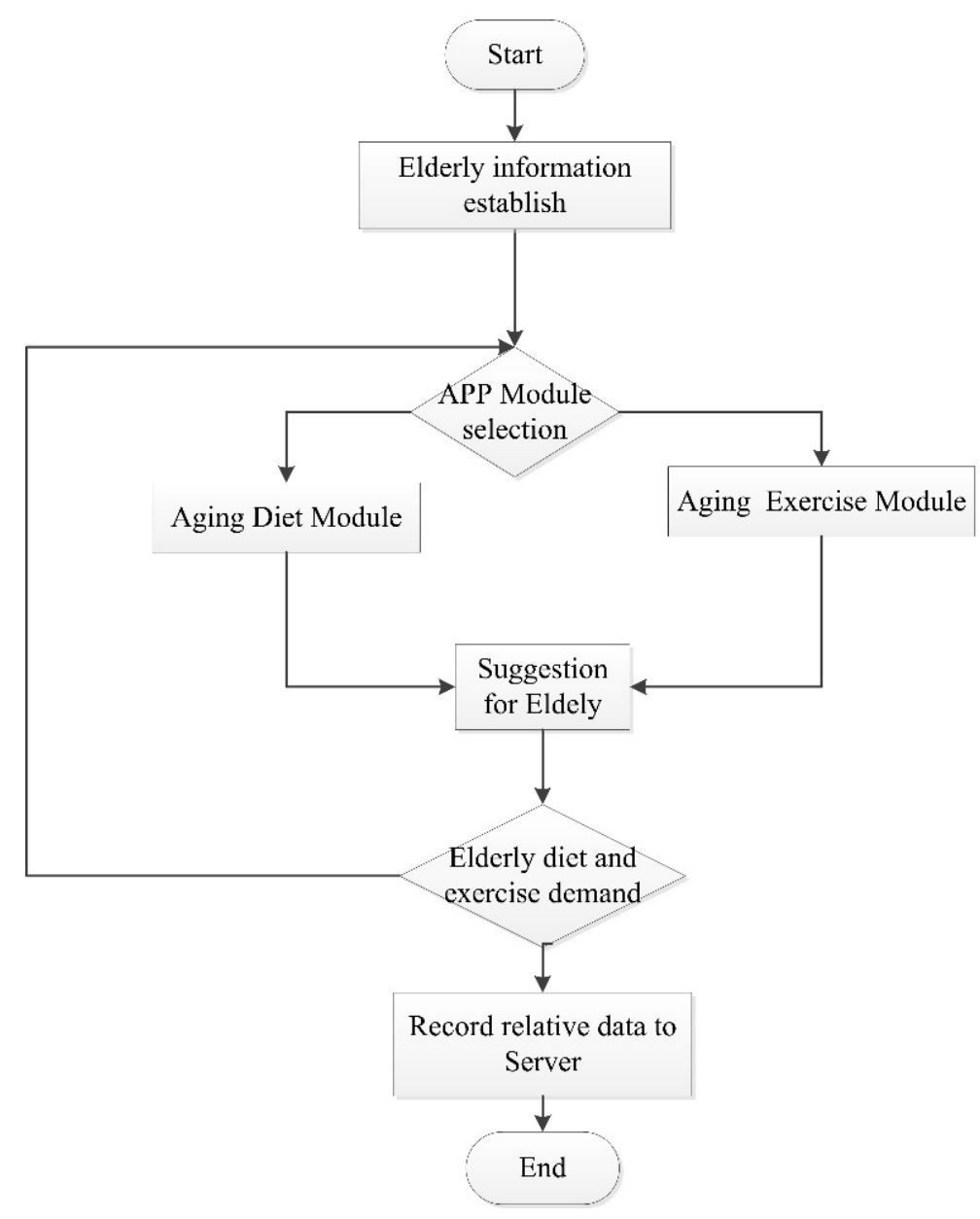

Figure 4. Flowchart for health promotion app.

1. Aging Diet Module: This module can be used to record the daily diet of the elderly and confirm whether the daily nutritional intake is balanced. It also provides timely nutritional dietary suggestions. This module provides healthy and nutritious diet recommendations based on easy eating, adequate calorie intake, and balanced nutrition (considering six categories of food). Taking a three-day diet as an example, the following recipe can be planned. The system provides elderly people with healthy and nutritious diet recommendations in a cycle of 3 days and provides a variety of different recipe combinations. This system can give suggestions based on the eating situation and extent of the elderly and will give diversified suggestions based on the personal information (such as chewing ability and degree of mobility) input by the user for the first time.

2. Aging Exercise Module: This module intends to promote health and maintain exercise. It adheres to the FITT exercise principle and gives corresponding exercise suggestions for the elderly, utilizing different body functions. The activity suggestions provided by this module for the 
elderly are as follows: (1) We have noticed that many elderly people who want to engage in sports have some degree of physical restrictions. It is necessary to recommend different exercise prescriptions according to their physical conditions, such as weight-bearing or non-weight-bearing and low or medium intensity. (2) Sports types such as pedaling, swimming, and using a chair and floor for sports are all appropriate choices. Pay attention to the safety, entertainment level, and feasibility of these sports. The most important thing is whether seniors can attach themselves to the movement and continue to interact with it. (3) The choice of exercise mode should pay attention to the individuality and the uniqueness of the elderly person. In addition to exercise stipulations, attention should also be paid to personal preferences. (4) The exercise prescription should also include modified stretching and muscle training.

\subsection{Discussion}

This system will be compared with temperature sensors combined with ZigBee technology for research in nursing, a wireless remote home health care system [20], and a wireless health monitoring system [21]. The items being compared are shown in the Table 2 below.

Table 2. Comparison of system function.

\begin{tabular}{cccc}
\hline System Function & The Proposed System & [21] & [20] \\
\hline Mobile User Interface & Yes & Yes & Yes \\
Real Time alert & Yes & Yes & Yes \\
Remote Monitor & Body temperature, heart & Body temperature, heart & Body temperature, \\
rate, breathing, oxygen & rate, breathing, oxygen & oxygen, blood pressure \\
Environment Monitor & Yes & No & No \\
Health Promotion & Yes & No \\
\hline
\end{tabular}

1. Platform interface: For the construction of the system platform, this system is designed through Visual Studio in Microsoft Visual Studio.NET. The system used in $[20,21]$ is designed by C\#.

2. Instant warnings: Instant warnings are presented in different ways in each system. Some will be notified by e-mail, some will be in the form of mobile text messages, and some will even make a sound or notify the major hospitals. The system of [20] is to send emergency notification messages to family members' mobile phones through reminders on the interface and using the Skype API. In [21], the system posted warnings via the Internet. In addition to providing warning-related text on the system interface, this system also uses the ZigBee function to send back warnings, sounds at the end of the user's measurement, and instantly informs the user.

- Remote monitoring: For the selected instrument, all three systems use ZigBee's wireless network to build a remote monitoring system. The choice of measured physiological signals is varied. In [21], the system uses body temperature, blood pressure, and blood oxygen. In [20], this system mainly measures body temperature, heart rate, breathing, and oxygen signals.

- Environmental monitoring: Since the user's living environment should be made more comfortable, this system has designed the function of environmental monitoring. Sensor chips are placed in the user's living room. The sensor chips send data to the computer system through the ZigBee network. With this design, users can understand the conditions in the room through the data on the computer system. Further, through the integration of the computer system and various electrical appliances, the room environment can be adjusted to a more comfortable situation.

- Health Promotion: This study integrates three major characteristics to construct a comprehensive home care system that is suitable for elderly people and has both context awareness and health promotion. Based on the Internet of Things technology and combined with wireless sensing 
networks, we hope to provide context awareness services for living scenarios-at the same time, providing exercise service modules for the elderly to promote health.

\section{Conclusions}

Most of the home care systems available on the market today are focused on health care and medical protection. The originality of this research includes the integration of the following three characteristics to build a comprehensive health-promoting system that is suitable for the elderly and has both context awareness and health promotion: (1) This study created a design based on IoT technology and a wireless sensing network. In addition to collecting the elderly's physiological information, it also uses sensors to obtain situational information. Through this scenario, the system can infer the services needed by the elderly and provide them to the users. Its services have four main characteristics: automation, inclusivity, immediacy, and customization. (2) This study also provided context awareness services for living scenarios. The information provided by elderly people relying on various awareness equipment in family living scenarios can provide personal preferred context awareness services to the living environment. (3) On a practical level, nutrition is an important key to promoting the health of the elderly. This study incorporates the concept of an elderly nutritional diet, targeting the nutritional status of the elderly and the nutritional needs of various geriatric syndromes, plus giving exclusive daily nutritional diet recommendations based on individual conditions. This system uses the Aging Diet Module to advise elderly people how to choose a nutritious diet. The Aging Exercise Module provides the recommended amount of exercise and content to the user and records the diet and activity history to the relevant caregiver for reference of subsequent care. In addition to obtain good health and medical care for the elderly, this system also improves the daily diet of the elderly, further promoting health and reducing the risk of illness and becoming bedridden. The health-promoting care system based on context awareness that is proposed in this paper can not only provide comprehensive and integrated care services for the elderly, but also improve the diet and health of the elderly and reduce the incidence of illness or becoming bedridden. It is expected to work to effectively influence, improve health care, reduce the burden on medical resources required by the elderly in Taiwan, and to enhance the quality of care for local elderly people. In sum, it is hoped that the needy can be properly cared for, and ultimately, the elderly will live a healthier and more comfortable life.

Author Contributions: C.-H.L. and J.-F.T. contributed equally to the conception of the idea, implementing and analyzing the experimental system, writing the manuscript, revision and proofreading of the manuscript. All authors have read and agreed to the published version of the manuscript.

Funding: This research received no external funding.

Conflicts of Interest: The authors declare no conflict of interest.

\section{References}

1. Strümpel, C.; Billings, J. Overview on Health Promotion for Older People. Available online: https: //core.ac.uk/download/pdf/13527.pdf (accessed on 28 October 2020).

2. Sowa, A.; Tobiasz-Adamczyk, B.; Topór-Mądry, R.; Golinowska, S. Health status of older people-Evidence in Europe. Public Health Gov. 2015, 13, 381-396.

3. Marengoni, A.; Angelman, S.; Melis, R.; Mangialasche, F.; Karp, A.; Gramen, A.; Meinow, B.; Fratiglioni, R. Aging with multimorbidity: A systematic review of the literature. Ageing Res. Rev. 2011, 10, 430-439. [CrossRef] [PubMed]

4. De Friese, G.H.; Ory, M.G. Self Care in Later Life; Springer: New York, NY, USA, 1998.

5. Akyildiz, F.; Su, W.; Sank, Y.; Cyirci, E. A Survey on Sensor Networks. IEEE Commun. Mag. 2002, 40, $102-114$. [CrossRef]

6. Tablado, A.; Illarramendi, A.; Baglies, M.I.; Bermudez, J.; Goni, A. Aingeru: An Innovating System for Tele-assistance of Elderly People; InTelecare: Porto, Portugal, 2004; pp. 27-36.

7. Tun, S.Y.Y.; Madanian, S.; Mirza, F. Internet of things (IoT) applications for elderly care: A reflective review. Aging Clin. Exp. Res. 2020, 10, 1-3. [CrossRef] [PubMed] 
8. Lamothe, L.; Fortin, J.P.; Labbe, F.; Gagnon, M.P.; Messikh, D. Impacts of Tele-homecare on Patients, Providers, and Organizations. Telemed. J. E-Health 2006, 12, 363-369. [CrossRef] [PubMed]

9. Filippo, P.; Jonas, U. Sensor network infrastructure for a home care monitoring system. Sensors 2014, 14, 3833-3860.

10. General Assembly Resolution 46/91 of 16 December 1991. Available online: https://www.ohchr.org/ Documents/ProfessionalInterest/olderpersons.pdf (accessed on 28 October 2020).

11. Gao, T.; Pesto, C. Wireless Medical Sensor Networks in Emergency Response: Implementation and Pilot Results. In Proceedings of the IEEE International Conference Technologies for Homeland Security, Waltham, MA, USA, 12-13 May 2008; pp. 187-192.

12. Tamilselvi, V.; Sribalaji, S.; Vigneshwaran, P.; Vinu, P.; GeethaRamani, J. IoT based health monitoring system. In Proceedings of the 2020 6th International Conference on Advanced Computing and Communication Systems (ICACCS), Singapore, 4-8 December 2017; pp. 386-389.

13. Acharya, A.D.; Patil, S.N. IoT based health care monitoring kit. In Proceedings of the 2020 Fourth international conference on computing methodologies and communication (ICCMC), Erode, India, 11-13 March 2020; pp. 363-368.

14. Islam Ayon, S.; Milon Islam, M. Diabetes prediction: A deep learning approach. Int. J. Inf. Eng. Electron. Bus. 2019, 11, 21-27. [CrossRef]

15. Schilit, B.N.; Theimer, M.M. Disseminating active map information to mobile hosts. IEEE Netw. 1994, 8, 22-32. [CrossRef]

16. Baumgartner, N. A tour of beaware-A situation awareness framework for control centers. Inf. Fusion 2014, 20, 155-173. [CrossRef]

17. Chen, J.; Kwong, K.; Chang, D.; Luk, J.; Bajcsy, R. Wearable Sensors for Reliable Fall Detection. Eng. Med. Biol. Soc. 2005, 11, 3551-3554.

18. Hoeger, W.W. Principles and Labs for Fitness and Wellness, 6th ed.; Belmont: Wadsworth, OH, USA, 2002.

19. Peungsuwan, P.; Sermcheep, P.; Harnmontree, P. The Effectiveness of Thai Exercise with Traditional Massage on the Pain, Walking Ability and QOL of older people with knee osteoarthritis: A randomized controlled trial in the community. J. Phys. Ther. Sci. 2014, 26, 139-144. [CrossRef] [PubMed]

20. Pattichis, C.S.; Kyriacou, E.; Voskarides, S.; Pattichis, M.S.; Istepanian, R.; Schizas, C.N. Wireless Telemedicine Systems: An Overview. IEEE Antennas Propag. Mag. 2002, 44, 143-153. [CrossRef]

21. Teaw, E.; Hou, G.; Gouzman, M.; Tang, K.W.; Kesluk, A.; Kane, M.; Farrell, J.A. Wireless Health Monitoring System. In Proceedings of the 2005 IEEE Conference on Information Acquisition, Hong Kong, China, 27 June-3 July 2005.

Publisher's Note: MDPI stays neutral with regard to jurisdictional claims in published maps and institutional affiliations.

(C) 2020 by the authors. Licensee MDPI, Basel, Switzerland. This article is an open access article distributed under the terms and conditions of the Creative Commons Attribution (CC BY) license (http://creativecommons.org/licenses/by/4.0/). 